\title{
Endometrial Dedifferentiated Carcinoma
}

National Cancer Institute

\section{Source}

National Cancer Institute. Endometrial Dedifferentiated Carcinoma. NCI Thesaurus. Code C126769.

A carcinoma that arises from the endometrium and is characterized by the presence of an undifferentiated carcinomatous component and a second component of either FIGO grade 1 or 2 endometrioid carcinoma. 